\title{
Where now with NICE?
}

\author{
E. M. Dennison • C. Cooper
}

Received: 17 December 2010 / Accepted: 17 December 2010 / Published online: 29 January 2011

(C) International Osteoporosis Foundation and National Osteoporosis Foundation 2011

\section{Introduction}

The National Institute of Clinical Excellence (NICE) is the agency in the UK, charged with the task of appraising Novel Health Technologies. Since its inception in 1999, the institute has frequently been mired in controversy. One recent example of this controversy is the divergence between established clinical practice for the management of osteoporosis, and the advice provided by NICE on this topic to health care purchasers $[1,2]$. This set of final appraisal documents has taken an astonishing 8 years to be completed. In the appraisals, intervention thresholds for primary prevention are based on a complex matrix of age, clinical risk factors and bone density specific for each agent that is used in the prevention of bone loss and fracture. The guidance decrees that a woman who fulfils criteria for therapy with alendronate, but is then unable to tolerate the agent, may have to wait until her bone density drops below a substantially lower threshold before she becomes eligible for other (marginally more expensive) therapies. As is often the case with a slowly moving review process, newer therapies have emerged even as other therapies remain under evaluation, so that guidance is now restricted to a subset of agents currently licensed for the treatment of postmenopausal osteoporosis.

Before NICE, the guidelines of the Royal College of Physicians were widely utilised in the UK [3, 4]. These suggested that the decision to initiate therapy be based largely on physician assessment of a range of clinical risk factors for

E. M. Dennison · C. Cooper $(\bowtie)$

MRC Lifecourse Epidemiology Unit, University of Southampton,

Southampton General Hospital,

Southampton SO16 6YD, UK

e-mail: cc@mrc.soton.ac.uk fracture, followed by a DXA scan, using the WHO threshold (a $T$ score of -2.5) as the marker for intervention. Over the previous two decades, clinicians have been inundated with studies suggesting that several risk factors might comprise indications for bone densitometry, and it was clear that some of these acted on fracture risk through an influence on bone mineral density (BMD), while others did not. In addition, some risk factors were amenable to modification (for example, intake of alcohol and smoking), whereas others, such as age and gender, were not. Finally, it was felt that meaningful dialogue between patient and physician was inhibited by difficulties in explaining the likelihood of fracture using the $T$ score, and that this also impacted adversely on adherence rates to osteoporosis medication (below $50 \%$ at 1 year). Thus, the traditional approach had become relatively ineffective and not sufficiently prescriptive about how to use the many available therapies.

In the intervening period between the Royal College of Physicians guidance and the appraisals provided by the NICE, the WHO supported development of a fracture risk assessment tool, which was completed in $2008\left(\right.$ FRAX $\left.^{\mathbb{B}}\right)$. The FRAX algorithm (http://www.shef.ac.uk/FRAX) uses a variety of clinical risk factors, easily assessed in clinical practice, with or without the addition of a BMD result, to compute the 10-year probability of fracture for an individual. From this, a clinician and patient can decide on the initiation of therapy. With the difficulties inherent in the NICE appraisals, and the emergence of the FRAX algorithm, a novel approach to osteoporosis care was proposed by the National Osteoporosis Guideline Group (NOGG) [5]. This incorporates the use of the FRAX algorithm, together with intervention thresholds validated but not driven by costutility analyses, to target therapy to patients.

In a recent issue of the Archives of Osteoporosis, Kanis and colleagues provide a detailed critique of the NICE guidance 
for the prevention of fragility fractures in postmenopausal women with osteoporosis, which highlights the practical difficulties it raises and concerns regarding the modelling employed [6]. They review the different guidance currently available in the UK, including that available from the Royal College of Physicians, FRAX and the NOGG. The review provides a detailed evaluation of the NICE appraisal, highlighting differences with the FRAX approach. In a review of the cost-effectiveness analysis performed for NOGG, they point out that the calculations were performed on the basis of an annual cost of $£ 95$ for generic alendronate-while the actual cost has now fallen by about $75 \%$. It is pointed out that if resources were allocated to osteoporosis, then this may allow innovative therapy - but in reality, the use of agents, other than alendronate in the UK, is in a minority and continues to fall.

The approach adopted by NICE was, of course, fundamentally different from that of NOGG; the guidance is restricted to postmenopausal women with a $T$ score of -2.5 or below, and other risk factors for fracture (excluding men and glucocorticoid-induced osteoporosis). NICE also distinguishes between primary and secondary prevention, weighting the latter higher. The approach adopted leads to the differing treatment thresholds described previously, and the difficulty with its adoption in clinical practice. The economic model adopted by NICE has been criticised, and these criticisms are rehearsed in the Kanis review, including a discussion of the selective failure to adopt the clinical risk factors included in FRAX, and the effect of the impact of risk factors on the death hazard. In the review, Kanis and colleagues go on to assess the impact of the use of FRAX and changing the assumptions surrounding the model on the cost-effectiveness of strontium. They provide cost-effectiveness scenarios for women with a prior fracture and osteopenia, and in opportunistically assessed women with a $T$ score of $-2.5 \mathrm{SD}$ or below and a clinical risk factor (except smoking), i.e. at very different thresholds for treatment compared to NICE.

In a recent paper, Bolland et al. [7] compared the approach favoured by NOGG with the US-based National Osteoporosis Foundation (NOF) guidance, based on a cohort of older women who participated in a 5-year randomised controlled trial of calcium supplementation and compared the treatment recommendations with fracture outcomes over 5 years for each algorithm. In their cohort, a total of 143 subjects (10\%) sustained a non-traumatic osteoporotic fracture, and 21 sustained a non-traumatic hip fracture (1.4\%). Applying the NOF guidelines required that $97 \%$ of participants undergo bone densitometry and $48 \%$ receive treatment. Seventy-six percent of hip fracture cases and $63 \%$ of osteoporotic fracture cases were identified for treatment. Applying the NOGG guidelines required that $13 \%$ of participants undergo bone densitometry and $21 \%$ receive treatment.

It is inevitable that cost-effectiveness models will become outdated as further therapies for osteoporosis become generic.
For example, the annual cost for a year's supply of generic alendronate was listed by NICE as $£ 53$ annually (it was $£ 95$ in the NOGG model and has, of course, dropped further), contrasting with an annual cost of risedronate therapy of about $£ 264$, and a corresponding figure for strontium of $£ 333$. The new agent, densoumab, has been priced competitively with these two agents. As drug patents expire, the horizon for osteoporosis prescribing is likely to change again.

In summary, most specialists feel that it is a combination of the varying thresholds for initiation with different osteoporosis therapies, and the lack of accommodation of FRAX-listed risk factors that has made the NICE guidance least amenable to use in clinical practice. Physicians struggle to interpret these differing thresholds for therapy, and patient groups are understandably vocal about the idea that a woman who is deemed eligible for alendronate therapy, but is unable to tolerate it, will have to wait for her disease to deteriorate before another therapy becomes available to her. The physician also struggles to find guidance for treatment of a woman with a prior fragility fracture but whose bone density $T$ score is above $-2.5 \mathrm{SD}$. The inclusion of FRAX on bone density printouts, and most recently, its appearance as an i-Phone application, is a marker of the readiness with which it has been taken up by the osteoporosis community, and it is to be hoped that as we work toward greater translatability between FRAX and NICE, we are about to enter a dawn of more effective policy for prevention and treatment.

\section{References}

1. National Institute for Health and Clinical Excellence (2010) Final appraisal determination 161. Alendronate, etidronate, risedronate, raloxifene, strontium ranelate and teriparatide for the secondary prevention of osteoporotic fragility fractures in postmenopausal women. NICE, London, December 2010

2. National Institute for Health and Clinical Excellence (2010) Final appraisal determination160. Alendronate, etidronate, risedronate, raloxifene and strontium ranelate for the primary prevention of osteoporotic fragility fractures in postmenopausal women. NICE, London, December 2010

3. Royal College of Physicians (1999) Osteoporosis: clinical guidelines for the prevention and treatment. Royal College of Physicians, London

4. Royal College of Physicians and Bone and Tooth Society of Great Britain (2000) Update on pharmacological interventions and an algorithm for management. Royal College of Physicians, London

5. Compston J, Cooper A, Cooper C, Francis R, Kanis JA, Marsh D, McCloskey EV, Reid DM, Selby P, Wilkins M, National Osteoporosis Guideline Group (NOGG) (2009) Guidelines for the diagnosis and management of osteoporosis in postmenopausal women and men from the age of 50 years in the UK. Maturitas 62:105-108

6. Kanis JA, McCloskey EV, Jonsson B et al (2010) An evaluation of the NICE guidance for the prevention of osteoporotic fragility fractures in postmenopausal women. Archives of Osteoporosis. doi:10.1007/s11657-010-0045-5

7. Bolland MJ, Grey A (2010) Disparate outcomes from applying U. K. and U.S. osteoporosis treatment guidelines. J Clin Endocrinol Metab 95:1856-1860 